\title{
Web 2.0 en los ciberdiarios de América Latina, España y Portugal
}

\author{
Por Santiago Tejedor-Calvo
}

\begin{abstract}
Resumen: Se analiza la presencia y el tipo de uso de las plataformas y recursos de diálogo de la web 2.0 en los principales ciberdiarios de Iberoamérica. Los resultados derivan de una reciente investigación aplicada a 70 medios de comunicación online de América Latina, España y Portugal con el objetivo de diagnosticar la presencia de la web social en estos medios, el tipo de aplicación de los recursos 2.0 y cómo éstos modifican el concepto de ciberdiario.

Palabras clave: Ciberperiodismo, Ciberdiario, Cibermedios, Medios online, Web 2.0,

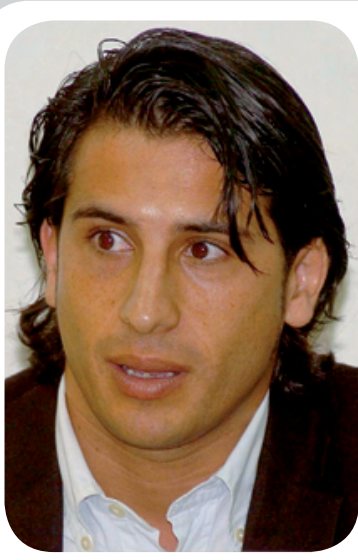

Santiago Tejedor-Calvo es licenciado en periodismo, máster de comunicación y educación, doctor en ingeniería de proyectos por la Universidad Politécnica de Catalunya (UPC) con la investigación "La web 2.0 en los ciberdiarios de Iberoamérica" y doctor en periodismo y ciencias de la comunicación por la Universidad Autónoma de Barcelona con la investigación "Estudio del ciberperiodismo en las universidades españolas". Es profesor de la Facultad de Ciencias de la Comunicación de la Universidad Autónoma de Barcelona (UAB), coordinador general de gabinete de Comunicación y Educación, coordinador del máster on-line de Comunicación, cultura y educación y coordinador del taller multimedia del Máster internacional de comunicación y educación. Autor del libro "La enseñanza del ciberperiodismo" (2007).
\end{abstract}

Periodismo participativo, Tecnología, Red, Internet.

Title: Web 2.0 in online newspapers of Latin America, Spain and Portugal

Abstract: The present article analyzes the presence and the type of use of the platforms and resources of the web 2.0 in the principal online newspapers of Latin America. The results derive from a recent study of 70 online mass media of Latin America, Spain and Portugal with the aim to diagnose the presence of the social web, the type of application of Web 2.0 resources and how these modify the concept of a web newspaper.

Keywords: Cyberjournalism, Cybermedia, Online journalism, Online newspapers, Web newspaper, Web 2.0, Technology, Network, Internet.

Tejedor-Calvo, Santiago. "Web 2.0 en los ciberdiarios de América Latina, España y Portugal”. El profesional de la información, 2010, noviembre-diciembre, v. 19, n. 6, pp. 610-619.

DOI: 10.3145/epi.2010.nov.07

\section{Web 2.0 y ciberdiario: una aproximación diagnóstica}

La web 2.0 o web social ha producido grandes cambios en el escenario comunicativo online, afectando a todas las plataformas y servicios de la red de redes en la medida en que introducen un componente destacado de horizontalidad, colaboración, creación conjunta y, especialmente, de participación.

En el caso de los medios de comunicación online las transformaciones son igualmente amplias y variadas ${ }^{1}$. Los medios han integrado en sus respectivas plataformas este conjunto de posibilidades de formas y con velocidades muy diversas. Hasta el momento, los foros, los chats y las encuestas habían absorbido prácticamente todo el protagonismo en la conformación de espacio de intercambio y diálogo dentro de los cibermedios $^{2}$. Este tipo de aplicaciones se caracteriza por contribuir a la formación y consolidación de "comuni- dades" virtuales de internautas vinculados por determinados intereses.

La web 2.0 y la filosofía que ésta conlleva han introducido nuevos espacios de intercambio donde el usuario asume nuevas facetas de comunicador, creador de contenidos e, incluso, validador de los mismos. En este sentido, un reciente estudio llevado a cabo por el Pew Research Center's Internet American Life Project y el Project for Excellence in Journalism, y centrado en EUA, muestra los hábitos de la audiencia de los medios y establece que las noticias hoy son "portátiles", "personalizadas" y "participativas".

http://pewinternet.org/

http://www.journalism.org/

Según la citada investigación:

- 33\% de los propietarios de teléfonos móviles accede a las noticias desde los mismos, 
- $28 \%$ de los usuarios de internet ha personalizado su página de inicio para incluir noticias de su interés,

- $37 \%$ contribuye a la creación de noticias, las comenta o las difunde en sitios como Facebook y Twitter.

Se añade que la fidelidad de la audiencia es cada vez más frágil. El 46\% de los usuarios afirma que consume noticias de cuatro a seis medios de comunicación. Apenas el 7\% obtiene información de una única fuente. Seis de cada diez estadounidenses (un 59\%) recibe noticias de una combinación de fuentes en línea y fuera de línea. La investigación sostiene que internet ha generado estos cambios en la audiencia y se indica que las redes sociales y los blogs han convertido el consumo de noticias en una experiencia social. La gente utiliza las redes sociales y la tecnología para filtrar, evaluar y reaccionar ante la información. Se destaca el crecimiento de la conectividad móvil mediante los smartphones, lo cual ha llevado a muchos a dar cuenta de los sucesos en tiempo real, como hemos visto en las recientes tragedias de Haití y Chile.

El trabajo citado introduce un conjunto de cambios de gran interés en la investigación de la comunicación online y, especialmente, sobre cómo la web 2.0 ha afectado a los diarios online (que tienen su origen hace poco más de una década, a principios de los años noventa).

Entre los investigadores que han trabajado en torno a la evolución del ciberperiodismo en el contexto iberoamericano, cabe señalar a Jesús Flores-Vivar y Guadalupe Aguado (2005) que apuntan un conjunto de rasgos del periodismo de internet estrechamente vinculados con las posibilidades comunicativas de la web 2.0 y su influencia en los ciberdiarios:

- Suma de tres medios: el periódico online se puede concebir como una fusión o suma de prensa, radio y televisión.

- Posibilidad de acceso a la información rápida y variada.

- Capacidad de actualización de la información de forma constante, sin límites de espacio y tiempo. A ello, se une la posibilidad de personalizar contenidos y de segmentar posibles mercados informativos.

- Eliminación de barreras geográficas en la cobertura informativa de la actualidad.

- Medio interactivo: el lector abandona su situación de pasividad en beneficio de un mayor protagonismo que le permite moderar, hasta cierto punto, el producto.

Por su parte, José-Luis Orihuela, profesor de la Universidad de Navarra, reflexiona, en una entrevista concedida al periodista João-Pedro Pereira sobre la web 2.0. Afirma que la llamada web 2.0 introduce una serie de herramientas de software social que han facilitado que "la gente corriente se comunique, coopere y publique de forma totalmente transparente". El autor apunta que la web social no es más que "la web de la gente, que es lo que siempre debió haber sido la web".

http://www.ecuaderno.com/2006/04/15/la-web-socialentrevista-en-diario-publico/

En este contexto, los cibermedios apuestan por nuevos espacios y aplicaciones dialógicas que promueven unos intercambios más variados y complejos que las tradicionales formas de apertura o participación, tal y como ya apuntaba, en los inicios del ciberperiodismo, Tanjev Schultz, del Institute for Intercultural and International Studies de la Universidad de Bremen, Alemania: "Tradicionalmente, el periodismo profesional ha ofrecido al público pocas oportunidades directas para la comunicación interactiva. Un medio muy conocido para la retroalimentación, especialmente en la prensa escrita, es la sección Cartas al director. Los lectores suelen escribir cartas como reacción a los mensajes de los medios. Sin embargo, la comunicación se queda aquí a menos que los periodistas u otros lectores (en cartas posteriores) respondan a la comunicación inicial. En principio lo mismo sucede con las llamas complementarias que se utilizan con frecuencia en programas de radio y televisión" (Schultz, 1999). En esta línea, el investigador y docente Javier Guallar refuerza estos planteamientos al destacar en uno de sus trabajos el incremento de espacio de interacción entre el medio, los contenidos y los usuarios a partir de la consolidación de la web 2.0. "Ahora se hace bien explícita la preocupación por hacer de los visitantes algo más que simples lectores, y se les invita ya no sólo a opinar sino además a participar en la presentación y la elaboración del contenido de los diarios. Los medios que realizan las actuaciones más decididas en esta línea se suman así a las tendencias que se extienden por la Red bajo la denominación web 2.0" (Guallar, 2007).

A ello se une la reflexión de Ainara Larrondo, profesora de la Universidad del País Vasco, que indica que en la información online es posible señalar tres rasgos que poseen un gran impacto e influencia en la representación de los mensajes y los géneros ciberperiodísticos. En todos ellos la web 2.0 potencia sus aplicaciones comunicativas: hipertextualidad e hipermedialidad; multimedialidad e interactividad. Larrondo destaca además otras señas de identidad del escenario comunicativo ciberperiodístico: temporalidad, memoria y personalización (Larrondo, 2008). Aunque la interacción entre ciudadanos y medios de comunicación en internet no es un hecho novedoso ya que hace años que éstos vienen ofreciendo los servicios denominados tradicionalmente de comunicación (foros, chats y encuestas), sí que es interesante destacar el avance parti- 
cipativo que se aprecia en la actualidad. Maxwell McCombs y un grupo de investigadores estadounidenses de la Universidad de Austin, Texas, que han llevado a cabo en los últimos años diferentes trabajos vinculados a los cibermedios hispanos en EUA y a iniciativas de comunicación online en México, destacan la importancia que la participación ciudadana que introduce la web 2.0 genera en el periodismo de internet: “¿Está todo esto -este periodismo ciudadano, la mezcla y fusión de aficionados y profesionales en la producción de noticias, esta aparente oportunidad para cualquier persona de informar e influir a escala masiva- realmente cambiando los medios? En nuestro ciclo de noticias en déficit de atención, "Bittergate" provocó un escándalo instantáneo, pero finalmente perdió su furor, cayendo con bastante rapidez de las cabeceras y de las agendas de los programas de radio de los domingos por la mañana, aunque sus brasas quemaran todavía durante semanas en la blogosfera hiper-partidista" (McCombs et al., 2010, p. 5).

Los investigadores Ernest Abadal y Javier Guallar, de la Universitat de Barcelona, agrupan los servicios de los diarios online en cuatro grandes categorías:

- Servicios de acceso a la información periodística de actualidad y retrospectiva: alertas por correo electrónico, móvil, RSS, consulta por hemerotecas, etc.

- Servicios referenciales: ayudan a resolver problemas de la vida cotidiana, como: clasificados, el tiempo, diccionarios, traductores, etc.

- Servicios de comunicación y participación: chats $\mathrm{y}$ foros.

- Servicios de ocio y entretenimiento: juegos y pasatiempos.

Esta clasificación se podría ampliar incorporando el conjunto de herramientas de la web 2.0. Resulta interesante observar que estos autores definen el diario actual como "multimedia social", ya que presenta un "incremento de los contenidos multimedia y [...] la potenciación de aspectos participativos de la web social" (Abadal; Guallar, 2010, p. 59).

Junto a los datos señalados, los planteamientos de Flores-Vivar y Aguado (2005), Orihuela (2006), Guallar (2007), Larrondo (2008), McCombs et al. (2010) y Abadal y Guallar (2010) entre otros, permiten afirmar que el ciberdiario encuentra en la web 2.0 un escenario de importantes novedades comunicativas que afectan directamente a las relaciones entre la plataforma, los mensajes, los ciberperiodistas y los usuarios. Incorporan un nuevo abanico de variables en los intercambios y sinergias informativas que se dan entre estos actores y componentes del esquema básico de la comunicación online.

\section{Objetivos y corpus de análisis de la investigación}

El presente artículo se basa en el análisis (cuantitativo y cualitativo) de las herramientas 2.0 que incorporan 70 diarios online de Iberoamérica (tabla 1). Se seleccionaron los de mayor prestigio, mejor posición en los rankings que estudian las visitas, más antigüedad o aquellos que han sido galardonados con algún reconocimiento internacional. Los datos se compilaron entre febrero de 2009 y febrero de 2010, durante tres días de la primera y tercera semana de cada mes.

También se realizaron consultas a cerca de 60 investigadores, ciberperiodistas y autores de reconocido prestigio en el marco de un estudio más amplio que realiza el autor sobre los cibermedios iberoamericanos.

La presente investigación intenta analizar cómo la web 2.0 afecta a los diarios online, y cómo éstos pueden aprovechar las posibilidades comunicativas $2.0 \mathrm{y}$ responder con un mayor grado de satisfacción a sus usuarios.

Se coincide en señalar que EUA es el país que ha llevado a cabo un mayor número de investigaciones sobre periodismo participativo desde diferentes enfoques y perspectivas. Tal y como apuntan Marcos Palacios y Javier Díaz-Noci (2006) la investigación empírica sobre el periodismo ciudadano es aún incipiente: "[...] las aproximaciones metodológicas son muy diversas, buscando la perspectiva más adecuada para abordar el fenómeno: análisis estructural de las oportunidades de participación que ofrecen las webs de medios convencionales, análisis de contenido de material 'periodístico' producido por los ciudadanos; entrevistas con periodistas, promotores de medios ciudadanos y ciudadanos mismos para entender sus actitudes y motivaciones".

Las tablas de recogida de datos se han organizado bajo los siguientes temas:

- Herramientas dialógicas: tipos, presencia y uso dentro del cibermedio.

- Referencia a los foros en la portada o home.

- Autonomía de los foros en el conjunto del cibermedio: si aparecen vinculados a un contenido o apartado o si, por el contrario, disponen de autonomía dentro de la estructura general.

- Referencia a los chats en la portada.

- Autonomía de los chats en el conjunto del cibermedio.

- Referencia a las encuestas en la portada.

- Autonomía de las encuestas en el conjunto del cibermedio.

- Referencia a las redes sociales en la portada. 


\begin{tabular}{|c|c|}
\hline País & Diario analizado \\
\hline España & $\begin{array}{l}\text { El país: http://www.elpais.com } \\
\text { La vanguardia: http://www.lavanguardia.es } \\
\text { El periódico: http://www.elperiodico.com } \\
\text { El mundo: http://www.elmundo.es }\end{array}$ \\
\hline Portugal & $\begin{array}{l}\text { Diário de notícias: http://www.dn.pt } \\
\text { Público: http://www.publico.pt } \\
\text { Jornal de notícias: http://www.jn.pt }\end{array}$ \\
\hline Argentina & $\begin{array}{l}\text { Clarín: http://www. clarin.com } \\
\text { Infonews: http://www.infonews.info } \\
\text { La nación: http://www. lanacion.com } \\
\text { Diario de Córdoba: http://www.cordoba.net } \\
\text { Diario crónica: http://www. diariocronica.com.ar/ }\end{array}$ \\
\hline Bolivia & $\begin{array}{l}\text { El diario: http://www.eldiario.net } \\
\text { El nuevo día: http://www.el-nuevodia.com } \\
\text { La razón: http://www.la-razon.com } \\
\text { El mundo: http://www.elmundo.com.bo } \\
\text { El potosí: http://elpotosi.net/2010/1004/ }\end{array}$ \\
\hline Brasil & $\begin{array}{l}\text { O globo: http://oglobo.globo.com/ } \\
\text { Folha de São Paulo: http://www.folha.uol.com.br } \\
\text { O estado de São Paulo: http://www.estadao.com.br }\end{array}$ \\
\hline Chile & $\begin{array}{l}\text { El mercurio: http://www.emol.com } \\
\text { La cuarta: http://www.lacuarta.cl } \\
\text { La nación: http://www.lanacion.cl } \\
\text { El sur: http://www.elsur.cl }\end{array}$ \\
\hline Colombia & $\begin{array}{l}\text { El espectador: http://www. elespectador.com } \\
\text { El tiempo: http://eltiempo.com. } \\
\text { La república: http://www.larepublica.com.co } \\
\text { El universal: http://www. eluniversal.com.co } \\
\text { El heraldo: http://www.elheraldo.com.co }\end{array}$ \\
\hline Costa Rica & $\begin{array}{l}\text { La nación: http://www.nacion.com } \\
\text { La prensa libre: http://www.prensalibre.co.cr/ } \\
\text { La república: http://www.larepublica.net }\end{array}$ \\
\hline Cuba & $\begin{array}{l}\text { El economista: http://www.eleconomista.cubaweb.cu } \\
\text { Granma: http://www.granma.cubaweb.cu } \\
\text { Juventud rebelde: http://www.juventudrebelde.cu }\end{array}$ \\
\hline Ecuador & $\begin{array}{l}\text { El comercio: http://www. elcomercio.com } \\
\text { El universo: http://www. eluniverso.com } \\
\text { Hoy: http://www.hoy.com.ec } \\
\text { El expreso: http://www.diario-expreso.com/ }\end{array}$ \\
\hline $\begin{array}{l}\text { EI } \\
\text { Salvador }\end{array}$ & $\begin{array}{l}\text { Diario coLatino: http://www. diariocolatino.com } \\
\text { Diario El Salvador: http://www. elsalvador.com }\end{array}$ \\
\hline Guatemala & $\begin{array}{l}\text { La hora: http://www. lahora.com.gt } \\
\text { Prensa libre: http://www. prensalibre.com.gt }\end{array}$ \\
\hline Honduras & $\begin{array}{l}\text { La prensa: http://www. Iaprensahn.com } \\
\text { La tribuna: http://www. Iatribuna.hn } \\
\text { Proceso digital: http://www.proceso.hn }\end{array}$ \\
\hline México & $\begin{array}{l}\text { El universal: http://www.el-universal.com.mx } \\
\text { La crónica de hoy: http://www.cronica.com.mx } \\
\text { Reforma: http://www.reforma.com } \\
\text { Multimedios: http://www.milenio.com }\end{array}$ \\
\hline Nicaragua & $\begin{array}{l}\text { El nuevo diario: http://www. elnuevodiario.com.ni } \\
\text { La prensa: http://www. laprensa.com.ni }\end{array}$ \\
\hline Panamá & $\begin{array}{l}\text { Panamá América: http://www.pa-digital.com.pa } \\
\text { El siglo: http://www.elsiglo.com }\end{array}$ \\
\hline Paraguay & $\begin{array}{l}\text { ABC digital: http://www.abc.com.py } \\
\text { Última hora: http://www.ultimahora.com }\end{array}$ \\
\hline Perú & $\begin{array}{l}\text { El comercio: http://www.elcomercioperu.com.pe } \\
\text { El sol: http://www.diarioelsol.com/ } \\
\text { Expresso: http://www.expreso.com.pe/ } \\
\text { Diario del Cusco: http://www.diariodelcusco.com }\end{array}$ \\
\hline $\begin{array}{l}\text { Puerto } \\
\text { Rico }\end{array}$ & $\begin{array}{l}\text { El nuevo día: http://www.endi.com/noticias } \\
\text { El vocero de Puerto Rico: http://www.vocero.com }\end{array}$ \\
\hline $\begin{array}{l}\text { República } \\
\text { Domini- } \\
\text { cana }\end{array}$ & $\begin{array}{l}\text { El Caribe CDN: http://elcaribe.com.do } \\
\text { Hoy: http://www.hoy.com.do } \\
\text { Listín diario: http://www.listindiario.com.do } \\
\text { Clave digital: http://www. clavedigital.com }\end{array}$ \\
\hline Uruguay & $\begin{array}{l}\text { El observador: http://www.observador.com.uy } \\
\text { El país: http://www.diarioelpais.com }\end{array}$ \\
\hline Venezuela & $\begin{array}{l}\text { El universal: http://www.eud.com } \\
\text { El mundo: http://www.el-nacional.com }\end{array}$ \\
\hline
\end{tabular}

Tabla 1. Listado de cibermedios analizados por países.
- Autonomía de las redes sociales en el conjunto del cibermedio.

- Redes sociales en los cibermedios: cuáles son las de mayor presencia.

- Referencia a los weblogs en la portada.

- Autonomía de los weblogs en el conjunto del cibermedio.

- Plataformas de creación y gestión de contenidos 2.0: tipos y características, precisando cuáles destacan.

- Apartado "Otros" dentro de las plataformas de creación y gestión de contenidos 2.0.

\section{- Principales contenidos 2.0.}

Se adjunta la tabla de recogida de datos utilizada y aplicada en el marco de la citada investigación (tabla 2).

\section{Aplicaciones dialógicas}

Las redes sociales (con una presencia del 38\%), las encuestas (32\%) y los foros (22\%) son los instrumentos de este tipo que presentan un mayor protagonismo. Los chats $(7 \%)$ tienen una presencia muy reducida, especialmente en comparación con las anteriores. Las redes sociales se sitúan como la herramienta más extendida dentro de esta categoría. Este aspecto constituye un dato relevante ya que (a diferencia de los foros, las encuestas y los chats) las redes sociales constituyen un tipo de plataforma propio de la web 2.0 que ha obtenido en este escenario un impulso muy amplio. Además este dato revela que en un tiempo reducido las redes sociales han pasado de ser sistemas dedicados a la formación de comunidades online a tener un protagonismo destacado dentro de los propios cibermedios, llegando incluso a asumir funciones de promoción, difusión e información (figura 1).

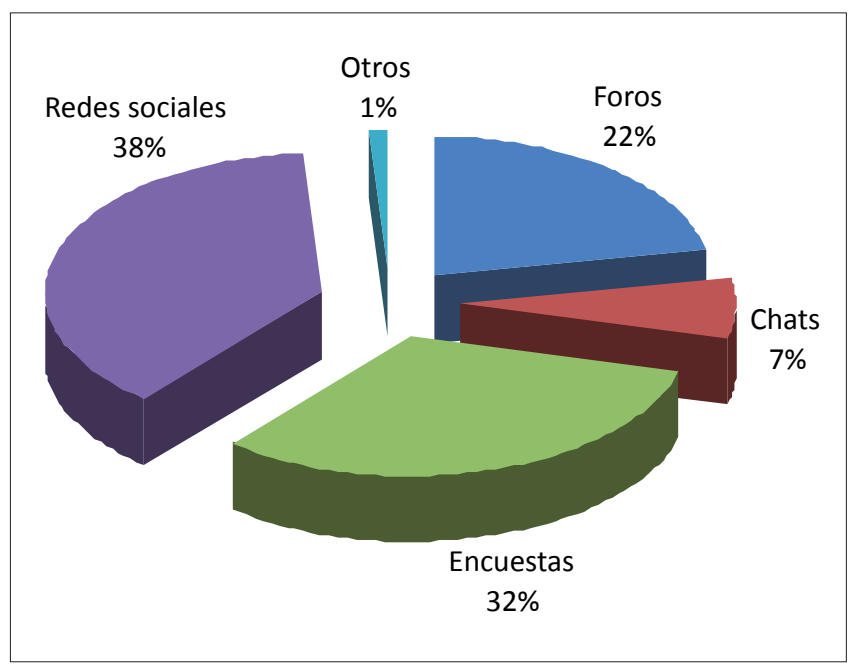

Figura 1. Herramientas dialógicas 


\begin{tabular}{|c|c|c|c|c|c|c|}
\hline \multicolumn{7}{|l|}{ Medio: } \\
\hline \multicolumn{7}{|l|}{ País: } \\
\hline \multicolumn{7}{|l|}{ Semana: } \\
\hline Recurso & Sí/ No & Número & Portada & $\begin{array}{l}\text { Dentro de } \\
\text { información }\end{array}$ & $\begin{array}{l}\text { Presencia } \\
\text { autónoma }\end{array}$ & $\begin{array}{c}\text { Otros } \\
\text { comentarios }\end{array}$ \\
\hline \multicolumn{7}{|c|}{ Herramientas dialógicas } \\
\hline \multicolumn{7}{|l|}{ Foros } \\
\hline \multicolumn{7}{|l|}{ Chats } \\
\hline \multicolumn{7}{|l|}{ Encuestas } \\
\hline \multicolumn{7}{|c|}{ Plataformas de creación y gestión de contenidos 20} \\
\hline \multicolumn{7}{|c|}{ Plataformas de creación y gestión de contenidos 2.0} \\
\hline \multicolumn{7}{|l|}{ Weblogs } \\
\hline \multicolumn{7}{|l|}{ Photoblogs } \\
\hline \multicolumn{7}{|l|}{ Audioblogs } \\
\hline \multicolumn{7}{|l|}{ Videoblogs } \\
\hline \multicolumn{7}{|l|}{ Wikis } \\
\hline \multicolumn{7}{|l|}{ Otros (especificar) } \\
\hline \multicolumn{7}{|c|}{ Contenidos 2.0} \\
\hline \multicolumn{7}{|l|}{ Mashups } \\
\hline \multicolumn{7}{|l|}{ Podcasts } \\
\hline \multicolumn{7}{|l|}{ Lectores RSS } \\
\hline \multicolumn{7}{|c|}{ Servicios para telefonía móvil } \\
\hline \multicolumn{7}{|l|}{ Otros (especificar) } \\
\hline \multicolumn{7}{|l|}{ Etiquetas o tags } \\
\hline \multicolumn{7}{|c|}{ Folksonomía (folksonomy) } \\
\hline \multicolumn{7}{|c|}{$\begin{array}{l}\text { Marcadores sociales (social } \\
\text { bookmarking) }\end{array}$} \\
\hline \multicolumn{7}{|c|}{ Otros (especificar) } \\
\hline \multicolumn{7}{|c|}{ Participación directa de los usuarios } \\
\hline \multicolumn{7}{|c|}{ Creación de "mi" cibermedio } \\
\hline \multicolumn{7}{|c|}{ Personalización de página de inicio } \\
\hline $\begin{array}{l}\text { Herramientas para } \\
\text { contenidos por los }\end{array}$ & & & & & & \\
\hline Periodismo ciudada & & & & & & \\
\hline Otros (especificar) & & & & & & \\
\hline & & os servici & e la web : & & & \\
\hline Redes sociales & & & & & & \\
\hline Buscadores 2.0 & & & & & & \\
\hline Otros (especificar) & & & & & & \\
\hline
\end{tabular}

Tabla 2. Uso de los recursos de la web 2.0

\section{"Los cibermedios iberoamericanos incorporan blogs como espacios autónomos, referenciados en portada y con autonomía temática"}

Por otro lado, se ha observado cómo los foros y muy especialmente los chats han experimentado un retroceso en su presencia dentro de los cibermedios. Este dato invita a la reflexión en torno al tipo de instrumentos y plataformas que priorizan los usuarios y cuáles de ellas consideran como más provechosas y/o interesantes en su proceso de consulta y navegación dentro de la estructura general de un periódico.

Un 55\% de los medios incluye alguna alusión a foros en su home, lo cual resulta llamativo.
Un 79\% de los que presentan foros les confieren una autonomía total, ocupando su propia sección o apartado dentro de la estructura general del cibermedio.

Un $44 \%$ de los medios que tienen chats lo indican en su home, y el 89\% de dichos medios les confieren también completa independencia. Este aspecto viene a subrayar la falta de sinergias entre diferentes instrumentos de la misma plataforma en la medida en que los chats, las encuestas, los foros, etc., serían recursos de utilidad para complementar las piezas informativas publicadas en las diferentes secciones del periódico.

Las encuestas tienen un gran peso dentro de la estructura general de los cibermedios puesto que las poseen el $74 \%$ en su página de inicio. De ellos, un $86 \%$ las incorpora con una autonomía total, esto es, no aparecen vinculadas a ninguna pieza informativa en concreto sino que ocupan un espacio propio (si bien se centran en algún aspecto relativo a la actualidad informativa). 


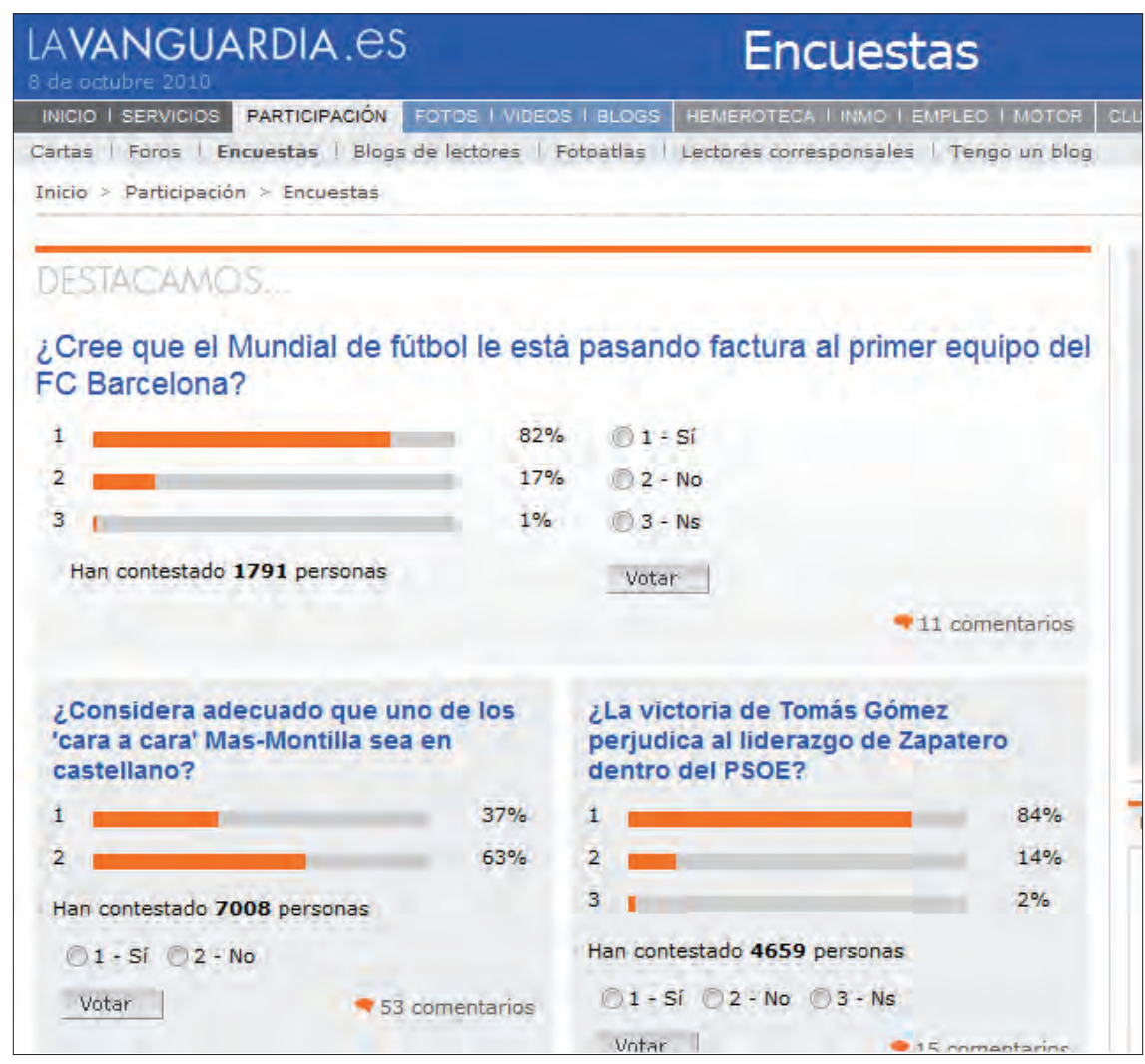

Encuestas en La vanguardia, http://www.lavanguardia.es/encuestas/index.html
Destaca el hecho de que un $60 \%$ de los periódicos que incorporan redes sociales les confieren una autonomía total. El 40\% restante vincula las redes sociales a algún tipo de contenido o apartado específico.

Respecto a las redes sociales, Facebook y el sistema de microblogging Twitter son los que disfrutan de una mayor presencia. MySpace, Orkut y $\mathrm{Hi} 5$ tienen un protagonismo muy reducido que se limita a uno o dos casos (figura 2).

\section{Weblogs}

Los weblogs son una de las herramientas que más se han extendido en los cibermedios en el escenario 2.0.

Un $83 \%$ de los analizados que presenta blogs incluyen alguna referencia, directa o indirecta, a éstos en sus respectivas portadas. O sea,

Incluso en ocasiones se ofrece al usuario la opción de acceder a un archivo histórico o fondo documental que recoge encuestas publicadas en fechas anteriores (precisando en cada caso el grado de participación y las opciones más votadas por los internautas).

\section{"La inclusión de herramientas 2.0 no garantiza la participación de los usuarios"}

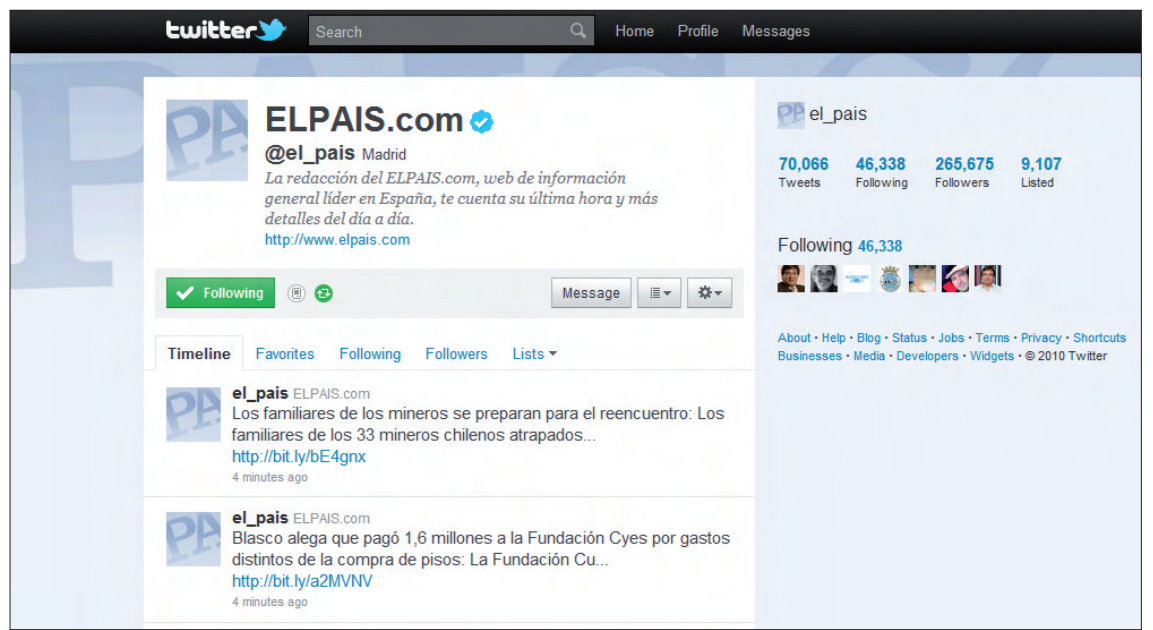

Twitter de El país, http://twitter.com/el_pais

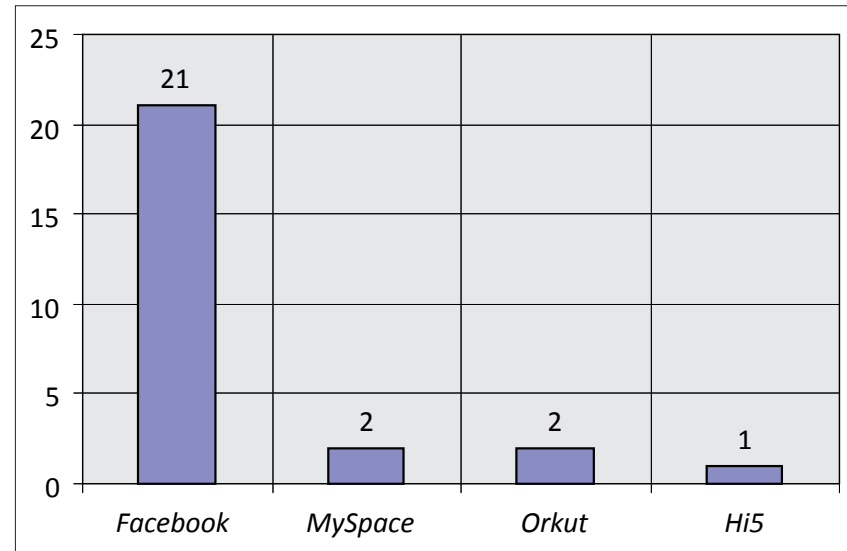

Figura 2. Redes sociales de los cibermedios 


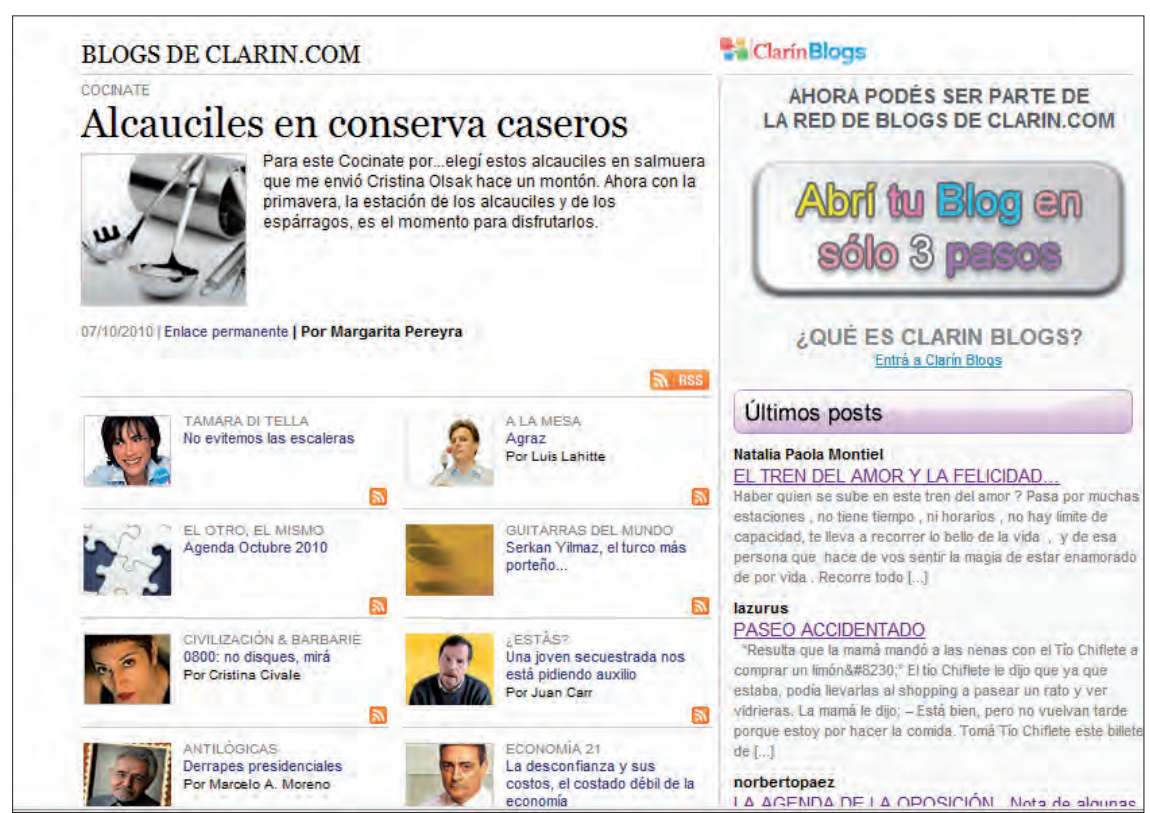

Blogs de Clarín, http://vww.clarin.com/weblogs/
$17 \%$ restante) el canal de publicación de vídeos YouTube (con un $67 \%$ ) y, por otro lado, de forma más reducida, otras plataformas como Yahoo (11\%), Flickr (11\%) y MyBlogLog (11\%) (figura 3).

La presencia destacada de Youtube en este apartado denota una amplia apuesta por los audiovisuales. Tal y como sucedía anteriormente con las redes sociales, este dato demuestra que los cibermedios, en el escenario de la web 2.0, han ampliado su alcance aprovechando plataformas e instrumentos propios de la red social que se caracterizan por su filosofía colaborativa, su planteamiento horizontal en el intercambio de contenidos, su gratuidad y su pluralidad tanto temática como de usuarios.

\section{Difusión y tratamiento de contenidos $\mathbf{2 . 0}$}

La presencia de contenidos 2.0 es uno de los aspectos de mayor interés en el marco del presente trabajo en la medida en que contribuye a establecer si los ciberdiarios han apostado por una transformación que, superando el aspecto estructural (esto es, la incorporación de nuevas plataformas) afecte a los mensajes que generan y que constituyen su principal referencia.

Los canales de sindicación de contenidos por RSS (con un 53\% sobre el total) tienen una presencia consolidada (figura 4).

Canal de Milenio en Youtube, http://www.youtube.com/milenio

donde se encuentran alojadas. Un 98\% les confieren un carácter autónomo dentro del conjunto, es decir, los conciben como una especie de micromedios dentro de la estructura general del medio principal. Generalmente son gestionados por expertos en diferentes materias o periodistas especializados en comentar la actualidad informativa. No obstante, los contenidos publicados en estos espacios no siguen de forma estricta la actualidad informativa sino que proponen sus propios temas y/o enfoques.

Dentro de las plataformas de creación y gestión de contenidos 2.0, más allá de los weblogs (que como se dijo abarcan un $83 \%$ del total), destacan (dentro del

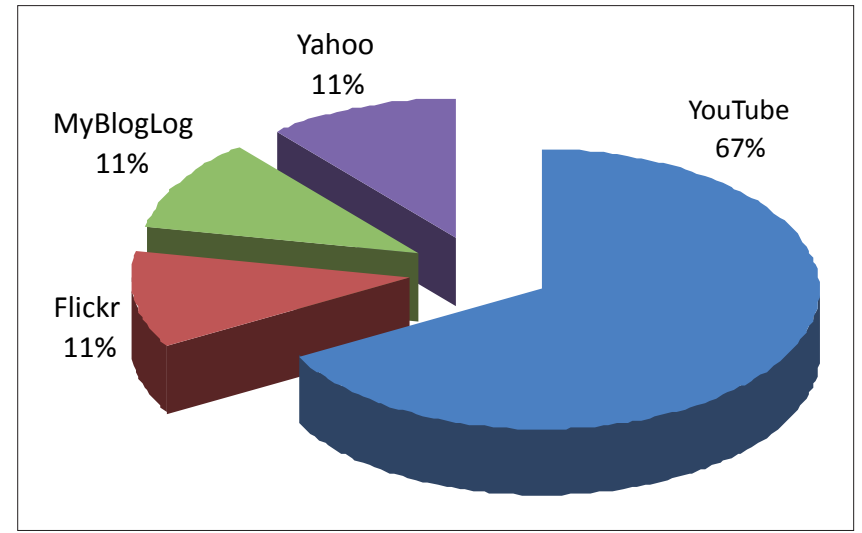

Figura 3. Apartado Otros: plataformas de creación y gestión de contenidos 2.0 


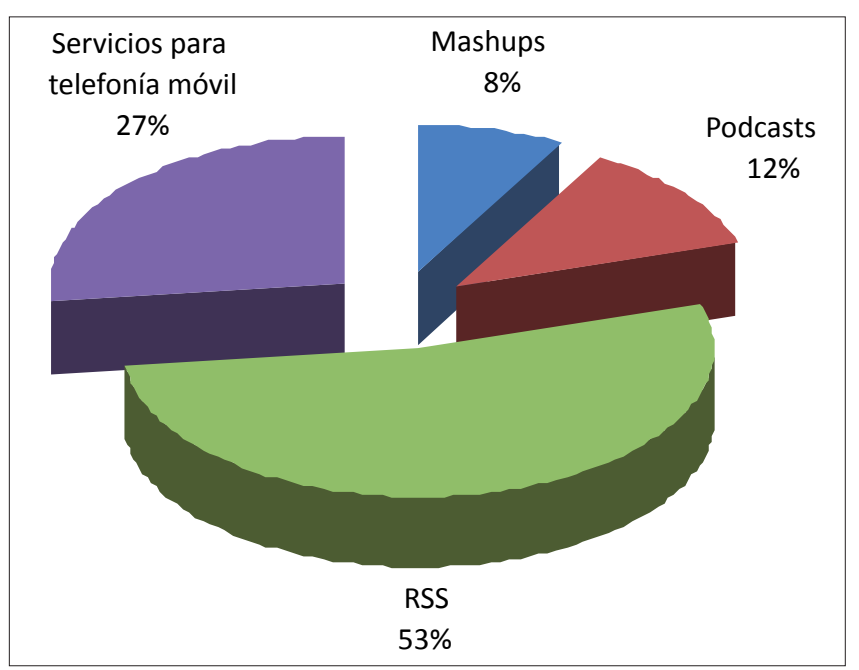

Figura 4. Principales contenidos 2.0

Los usuarios pueden "arrastrar" hasta sus propias plataformas (espacio de correo electrónico, weblogs, redes sociales, etc.) los contenidos de uno o múltiples medios mediante los canales de RSS. Además, esta sindicación de contenidos se puede realizar utilizando la tradicional estructuración de los contenidos en secciones (Política, Cultura, Sociedad, Deportes, Tecnología, etc.) o mediante otras opciones (Todos los titulares, Noticias más recientes, Toda la portada, etc.) que no responden a esa tradicional organización de la información. Los servicios de telefonía móvil (27\%) ocupan un lugar destacado. Sin embargo, en este caso, si bien la web 2.0 los ha potenciado, no podemos considerar que representen exactamente las características definitorias de la web social. Los podcasts (12\%) presentan una inclusión escasa. La combinación de plataformas y contenidos de diferentes espacios online, esto es, los mashups (8\%) son aún muy poco utilizados. Con relación a los podcasts y a los mashups es importante apuntar que, generalmente, están presentes en los medios que han llevado a cabo una apuesta más amplia y variada por los recursos de la web 2.0 (Tejedor-Calvo, 2008).

\section{Conclusiones}

El estudio diagnóstico sobre la presencia de los principales recursos dialógicos de la web 2.0 en los ciberdiarios de Iberoamérica permite apuntar que se encuentran sumidos en un importante proceso de transformación que afecta al conjunto de sus dinámicas de producción periodística. En el marco de este estadio de cambios, los espacios de carácter dialógico han cobrado una importancia destacada, reforzando el carácter horizontal y participativo que impulsa la web 2.0.

La tendencia apunta hacia un incremento de los espacios de comunicación, ocupando las redes sociales un puesto destacado, especialmente, Facebook y Twitter. Por su parte, las encuestas mantienen una presen- cia consolidada como herramienta de participación y apertura del ciberdiario hacia sus usuarios. Respecto a los chats y los foros, se observa una reducción de su protagonismo.

En sus portadas muestran enlaces a su espacios dialógicos (tanto redes sociales, como chats, foros y encuestas), aspecto que podría interpretarse como un esfuerzo por transmitir a los internautas una voluntad de horizontalidad y apuesta por la participación. No obstante, la inclusión de este tipo de herramientas y/ o espacios no puede concebirse como una garantía de apertura o participación. En numerosas ocasiones el diario no aporta ningún feedback o retroalimentación a los comentarios, intervenciones y propuestas de los usuarios.

En la mayoría de casos la incorporación de estos espacios de diálogo se realiza confiriéndoles una clara autonomía respecto al resto de secciones y contenidos. De este modo no se establece ninguna sinergia entre la herramienta dialógica y los contenidos informativos del ciberdiario. Por tanto, se desaprovecha claramente la posibilidad de generar contenidos online de carácter informativo enriquecidos con estas "vías" de diálogo.

Presentan una apuesta clara y amplia por la inclusión de weblogs o bitácoras on-line, y la práctica totalidad de los que los tienen aluden a ellos en su página de inicio. Es necesario, no obstante, apuntar que en un 98\% de los casos se trata de espacios autónomos aislados del resto de secciones y apartados. La inclusión de los blogs se podría equiparar con la creación de una especie de micro-medio dentro la estructura general del medio. En ocasiones, los weblogs se centran en los temas planteados en la portada, pero en la mayoría de casos tratan asuntos desvinculados. Se detecta de nuevo, pues, la falta generalizada de un esfuerzo por establecer dinámicas de convergencia y aprovechamiento mutuo entre los diferentes espacios del medio online.

\section{"La incorporación de contenidos sindicados por RSS ha experimentado un gran crecimiento"}

Con relación a los contenidos 2.0, cabe señalar que la incorporación de contenidos sindicados por RSS es la más amplia, seguida de los servicios para telefonía móvil, archivos sonoros descargables o podcasts, y seguidos por la presencia de mashups periodísticos, esto es, contenidos que integran diferentes aplicaciones generando un nuevo producto con una finalidad informativa. Esta inclusión destacada de RSS denota 


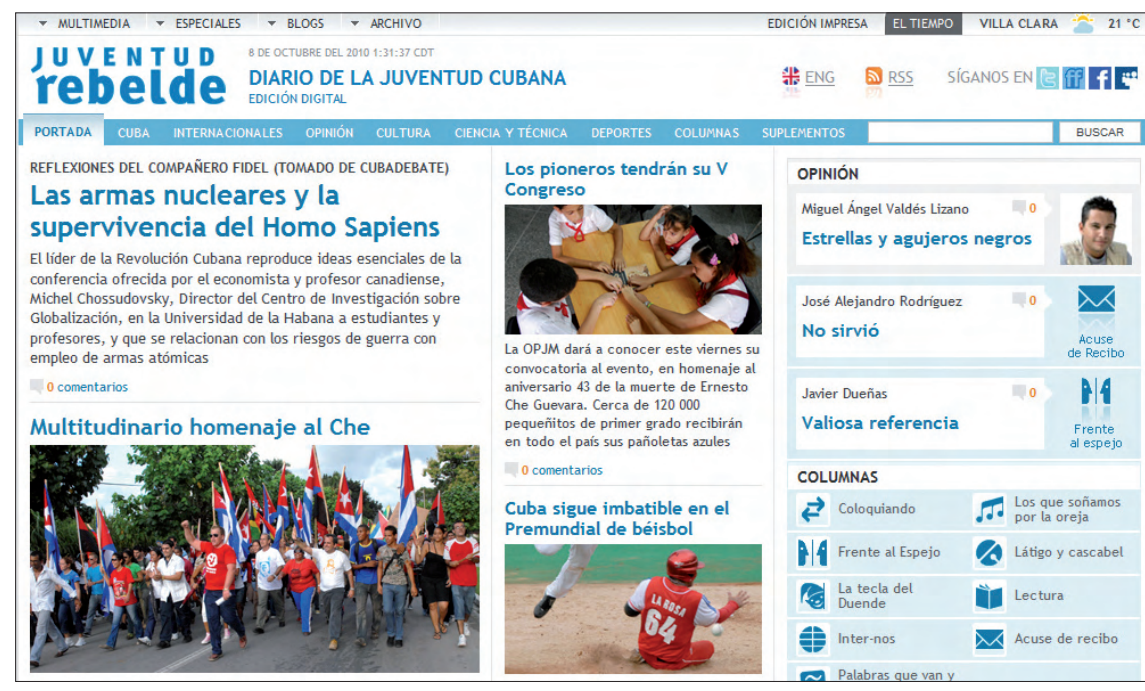

Juventud rebelde, http://www.juventudrebelde.cu

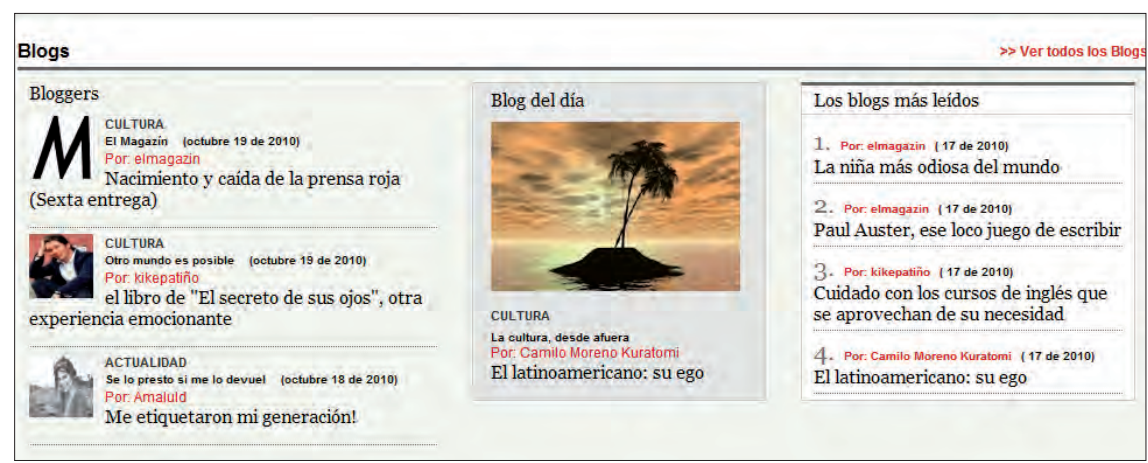

Blogs de El espectador, http://blogs.elespectador.com/ http://www.elpais.com

http://www.lavanguardia.es

http://www.clarin.com

http://www.elespectador.com

http://www.milenio.com

http://www.juventudrebelde.cu

\section{Notas}

1. El profesor e investigador de la Universidad Austral de Buenos Aires, Argentina, Roberto Igarza reflexiona sobre los diferentes niveles de participación que pueden detectarse en los cibermedios y señala: "Existen varios tipos de periodismo participativo. Se los puede clasificar según el nivel de compromiso de los participantes, entre un nivel de compromiso superficial, caracterizado por una participación que consiste en hacer comentarios a los contenidos publicados, y un nivel alto de compromiso, correspondiente a una participación dominante en la producción de contenidos" (Igarza, 2008, p. 198).

2. Los investigadores Pau Bolaños, de la Universidad Ramon Llull, y Manuel López, de la Universidad Autónoma de Barcelona, coinciden en destacar la importancia de este tipo de géneros dialógicos: "El sistema funciona de forma muy sencilla: alguien propone un tema genérico de debate desde su plataforma en la Red y alguien desde cualquier otro punto de la Red contesta en función del interés que le despierta el tema planteado. Se crea, pues, un foro de discusión en el cual el tiempo y el espacio adquieren fuerza un importante cambio que afecta a las facetas de producción y distribución de los mensajes en la medida en que, gracias a la sindicación de contenidos, el periódico puede alcanzar una mayor difusión de sus mensajes en diferentes plataformas de redes sociales, blogs, escritorios virtuales, etc.

\section{"Los diarios no aprovechan la sinergia que podría producirse entre sus contenidos y las aportaciones de los lectores"}

Los cibermedios que destacan por la aplicación amplia y variada de recursos 2.0 son: El país y La vanguardia en España; Clarín en Argentina; El espectador en Colombia; y Multimedios, en México. Por otro lado, aunque no puede considerarse como uno de los que mejor explotan las posibilidades de la web 2.0, el cibermedio cubano Juventud rebelde es interesante, ya que teniendo en cuenta la coyuntura socio-política de Cuba, incluye en su portada enlaces a diferentes espacios dialógicos y colaborativos de la web social, así como a varias redes sociales. o la pierden en función de los intereses de los colectivos. Los foros han resultado básicos para la creación de comunidades virtuales. [...] Los medios de comunicación con presencia en la Red entendieron que favorecer la participación de las audiencias en sus contenidos podía ser una forma de mejorar el conocimiento de las necesidades de sus audiencias. Sin embarexisten esos espacios de discusión, no podemos saber y ni siquiera intuir si realmente la opinión de los interactores influye sobre la agenda mediática o sobre el contenido de los medios. [...] Los foros proponen una nueva manera de cohesionarse socialmente al entorno de identidades múltiples, compartidas y de alguna manera reconstituidas" (Díaz-Noci; Salaverría, 2003, pp. 519-520).

3. El Bittergate es el nombre de un pequeño escándalo protagonizado por Barack Obama en abril de 2008, durante la campaña de las primarias, cuando, opinando sobre las personas en ciudades pequeñas de la América profunda, donde habían desaparecido puestos de trabajo, dijo: "Ellos se amargan (de ahí bitter), se aferran a las armas o a la religión o la antipatía hacia las personas que no son como ellos o el sentimiento anti-inmigrante o el sentimiento anti-comercio como una forma de explicar sus frustraciones".

\section{Webs}

\section{Wikipedia}

http://www.wikipedia.org

Facebook

http://www.facebook.com

MySpace

http://www.myspace.com

Hi5

http://www.hi5.com

Orkut

http://www.orkut.com go, y a pesar de que en todos los sitios web relacionados con los medios 
Twitter

http://www.twitter.com

Pew Research Center's Internet American Life Project"

http://pewinternet.org/

Project for Excellence in Journalism

http://www.journalism.org/

\section{Bibliografía citada}

Abadal, Ernest; Guallar, Javier. Prensa digital y bibliotecas. Gijón: Trea, 2010

Díaz-Noci, Javier; Salaverría, Ramón. (coords.). Manual de redacción ciberperiodística. Barcelona: Ariel, 2003.

Flores-Vívar, Jesús; Aguado, Guadalupe. Modelos de negocio en el ciberperiodismo. Madrid: Fragua, 2005.

Guallar, Javier. "La renovación de los diarios digitales: rediseños y web 2.0". El profesional de la información, 2007, mayo-junio, v. 16, n. 3, pp 235-242.

http://www.elprofesionaldelainformacion.com/contenidos/2007/mayo/08.pdf

Igarza, Roberto. Nuevos medios: estrategias de convergencia. Buenos Aires: La Crujía Ediciones, 2008

Larrondo, Ainara. Los géneros en la redacción periodística. Bilbao: Universidad del País Vasco, 2008.

McCombs, Maxwell; Willard-Hinsley, Amber; Kaufhold, Kelly; Lewis, Seth. The future of news. An agenda of perspectives. San Diego, EEUU: Cognella, 2010.
O'Reilly, Tim. What is web 2.0. Design patterns and business models for the next generation of software, 09-30-2005.

http://oreilly.com/web2/archive/what-is-web-20.html

Orihuela, José-Luis. La revolución de los blogs. Cuando las bitácoras se convirtieron en el medio de comunicación de la gente. Madrid: La esfera de los libros, 2006

Palacios, Marcos; Díaz-Noci, Javier (eds.). Ciberperiodismo: métodos de investigación, 2006.

http://www.argitalpenak.ehu.es/p291-content/es/contenidos/libro/se_indice_ciencinfoles_ciencinf/adjuntos/ciberperiodismo.pdf

Schultz, Tanjev. "Interactive options in online journalism: a content analysis of 100 U.S. newspapers". JCMC, Journal of computer-mediated communication, 1999. v. 5, n. 1

http://jcmc.indiana.edu/vol5/issue1/schultz.htm

Tejedor-Calvo, Santiago. "Periodismo 'mashup': combinación de recursos de la web social con una finalidad ciberperiodística". Revista anàlisi. 2008 , n. 35

Santiago Tejedor-Calvo. Departamento de Periodismo, Facultad de Ciencias de la Comunicación, Universidad Autónoma de Barcelona.

Campus UAB, Cerdanyola del Vallés, 08193 Bellaterra (Barcelona).

santiago.tejedor@uab.es

\section{ProQuest}

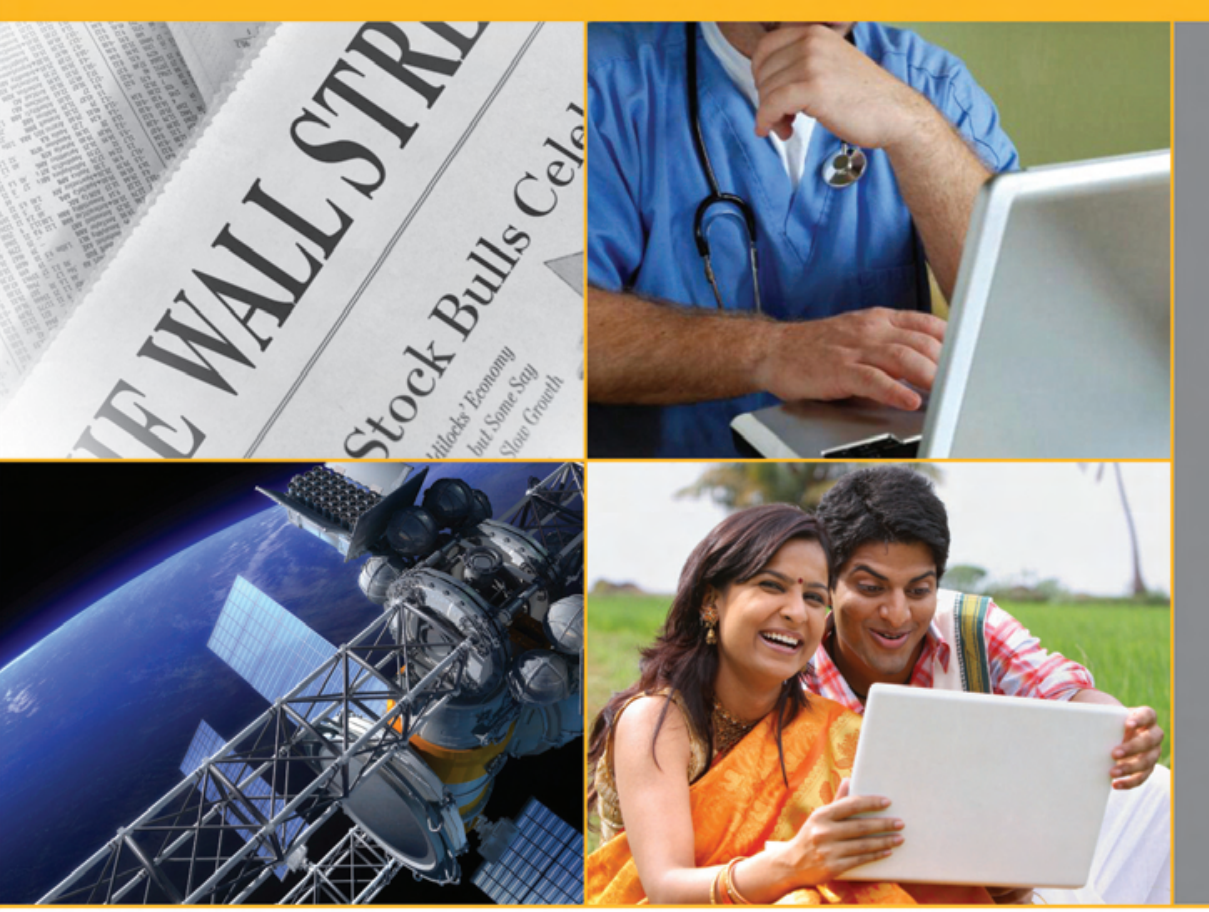

Contenido internacional en más de 160 áreas temáticas disponible desde un único punto de acceso. Una base de datos que cubre todas la necesidades de investigación.

- Más de 17.000 publicaciones periódicas incluyendo: The Lancet; MIT Sloan Management Review; Nature; Journal of Business Ethics; etc.

- Más de 700 periódicos internacionales a texto completo: The Guardian; The Times; The Wall Street Journal; 14 Financial Times titles; The Hindu; China Daily; etc.

Miles de tesis doctorales y documentos de trabajo (SSRN).

- Informes sobre sectores industriales e investigación de mercados: Business Monitor International (BMI), Economist Intelligence Unit (EIU), Dunn and Bradstreet/Hoover's, etc.

- Contenido profesional y herramientas de formación y enseñanza para investigadores. 